\title{
Plantas nativas da região amazônica: uma revisão integrativa acerca da sua
}

\section{aplicação na fitoterapia}

\author{
Native plants from the Amazon region: an integrative review of their application in herbal medicine
}

Plantas nativas de la región amazónica: una revisión integradora de su aplicación en la medicina

herbal

Recebido: 26/10/2021 | Revisado: 31/10/2021 | Aceito: 01/11/2021 | Publicado: 02/11/2021

Keliene Pereira

ORCID: https://orcid.org/0000-0002-4944-0889 Universidade Nilton Lins, Brasil E-mail: 17011793@uniniltonlins.edu.br

Maria Alves de Lima

ORCID: https://orcid.org/0000-0001-7328-0937 Universidade Nilton Lins, Brasil E-mail: 18006775@uniniltonlins.edu.br

Gabriel Oliveira de Souza

ORCID: https://orcid.org/0000-0001-9168-074X Universidade Nilton Lins, Brasil

E-mail: gabriel.souza@uniniltonlins.edu.br

\begin{abstract}
Resumo
O uso das plantas medicinais é uma prática milenar, fazendo parte da evolução humana, sendo consideradas os primeiros recursos terapêuticos da sociedade devido ao seu potencial farmacológico. Diante deste contexto o presente estudo buscou efetuar o levantamento etnobotanico sobre o conhecimento e uso das plantas medicinais da Amazônia, demonstrando a importância das plantas regionais com ação farmacológica para população. Sendo realizada uma pesquisa de fonte documental, com revisão integrativa da literatura, por meio de artigos publicados a respeito do tema em questão. Com base na análise e discussão dos resultados os estudos revelam a importância do uso de plantas medicinais mesmo em ambientes com poder aquisitivo alto, visando obter maiores estudos em torno das plantas medicinais da região amazônica que possuem ação farmacológica este trabalho através de bases de dados e uso de descritores, utilizou 20 artigos para uma revisão integrativa do tema, montando-se um quadro sinóptico com os principais resultados da revisão integrativa. É importante ressaltar que o uso de plantas medicinais ainda é um importante recurso utilizado para a manutenção da saúde de muitas comunidades carentes, onde são utilizadas para tratamentos comuns de saúde, tendo em vista que o custo é mais acessível à população, em comparação aos medicamentos obtidos por síntese química, que são em geral mais caros, devido às patentes tecnológicas envolvidas.

Palavras-chave: Fitoterapia; Plantas medicinais; Fitoterápicos.
\end{abstract}

\begin{abstract}
The use of medicinal plants is an ancient practice, being part of human evolution, being considered the first therapeutic resources in society due to their pharmacological potential. Given this context, this study sought to carry out an ethnobotanical survey on the knowledge and use of medicinal plants in the Amazon, demonstrating the importance of regional plants with pharmacological action for the population. Documentary source research was carried out, with an integrative literature review, through articles published on the topic in question. Based on the analysis and discussion of the results, the studies reveal the importance of using medicinal plants even in environments with high purchasing power, aiming to obtain further studies on medicinal plants from the Amazon region that have pharmacological action, this work through databases and Using descriptors, 20 articles were used for an integrative review of the topic, setting up a synoptic table with the main results of the integrative review. It is important to emphasize that the use of medicinal plants is still an important resource used to maintain the health of many poor communities, where they are used for common health treatments, considering that the cost is more accessible to the population, compared to medicines obtained by chemical synthesis, which are generally more expensive due to the technological patents involved.
\end{abstract}

Keywords: Phytotherapy; Medicinal plants; Herbal medicines. 


\section{Resumen}

El uso de plantas medicinales es una práctica milenaria, formando parte de la evolución humana, siendo considerados los primeros recursos terapéuticos en la sociedad por su potencial farmacológico. Ante este contexto, el presente estudio buscó realizar un relevamiento etnobotánico sobre el conocimiento y uso de plantas medicinales en la Amazonía, demostrando la importancia de las plantas regionales con acción farmacológica para la población. Se realizó una búsqueda de fuente documental, con una revisión integradora de la literatura, a través de artículos publicados sobre el tema en cuestión. A partir del análisis y discusión de los resultados, los estudios revelan la importancia del uso de plantas medicinales incluso en ambientes de alto poder adquisitivo, con el objetivo de obtener más estudios sobre plantas medicinales de la región amazónica que tengan acción farmacológica, este trabajo a través de bases de datos y utilizando descriptores, se utilizaron 20 artículos para una revisión integradora del tema, configurando una tabla sinóptica con los principales resultados de la revisión integradora. Es importante enfatizar que el uso de plantas medicinales sigue siendo un recurso importante para mantener la salud de muchas comunidades pobres, donde se utilizan para tratamientos de salud comunes, considerando que el costo es más accesible para la población, en comparación con los medicamentos obtenidos. por síntesis química, que generalmente son más caras debido a las patentes tecnológicas involucradas.

Palabras clave: Fitoterapia; Plantas medicinales; Hierbas medicinales.

\section{Introdução}

O uso das plantas medicinais é uma prática milenar, fazendo parte da evolução humana, sendo considerada o primeiro recurso terapêutico da sociedade devido ao seu potencial farmacológico, o que vem impulsionado cada vez mais o mercado farmacêutico ao longo dos anos. Assim, a fitoterapia que é o estudo das plantas medicinais e suas aplicações na terapia das doenças, é a forma mais antiga e fundamental de medicina da Terra. Assim, a fitoterapia permite ao homem se conectar com a natureza, passando a estudar as propriedades farmacológicas das plantas ou qualquer outro vegetal que apresente atividade biológica, ou seja, que podem ser usados para tratamento curativo de determinadas doenças, visando valorizar o conhecimento popular e validá-lo por meio de pesquisas (Abílio, 2011).

Dessa maneira geral, os estudos das plantas medicinais têm um papel fundamental, na propagação e conservação de determinados saberes tradicionais, pois constituem uma alternativa para tratar diversas enfermidades que por conta da situação de social e da falta de acesso a medicamentos industrializados, muitas comunidades dependem, essencialmente de plantas para os primeiros cuidados de saúde (Dantas \& Torres, 2019).

Diante da necessidade de incentivar o uso plantas medicinais nativas da região amazônica paro o tratamento e cura de doenças de uma forma mais simples, fácil e de baixo custo, (Feitosa et al., 2016) o presente estudo buscou resgatar o uso correto de plantas medicinais e o acesso a outras opções de tratamentos seguros e eficazes, por conta da imensa biodiversidade que floresta amazônica proporciona em recursos naturais (Silva et al., 2019).

Deste modo, a justificativa do presente estudo está baseada na busca de mais conhecimento em torno do assunto exposto, tendo em vista a importância dos medicamentos fitoterápicos e plantas medicinais regionais, visando destacar como plantas nativas da região amazônica podem ter ação terapêutica para os mais variados sistemas tais como: sistema respiratório, digestivo, neurológico, dermatológico e gástrico com suas respectivas descrições, proporcionando aos acadêmicos de saúde a população em geral a investigação das características estruturais, propriedades medicinais, princípios ativos, além de incentivar o uso correto e racional desse recurso natural (Vieira et al., 2010).

Pelo exposto, é de suma importância a promoção da conservação de saberes tradicionais em fitoterapia das plantas nativas da região amazônica, de seus benefícios e propriedades farmacológicas (Zeni et al., 2017). 


\section{Metodologia}

Visando alcançar o objetivo proposto neste trabalho, executou-se uma revisão integrativa da literatura, por meio de artigos publicados a respeito do tema em questão, tal método viabiliza a análise de pesquisas científicas de modo sistemático, amplo e favorece a caracterização da divulgação do conhecimento produzido. A busca online foi realizada por meio da Biblioteca Virtual em Saúde (BVS, DeCS) e Biblioteca Nacional de Medicina dos EUA (PubMed) na base de dados eletrônica Scientific Electronic Library Online (SciELO), Literatura Latino-americana e do Caribe em Ciências da Saúde (LILACS), e Sistema Online de Busca e Análise de Literatura Médica (MEDLINE).

Para operacionalizar a busca, utilizaram-se as palavras-chaves Fitoterapia; Plantas medicinais; Fitoterápicos. A partir dessa associação, foi feita a seleção dos artigos, sendo sua elaboração feita por meio do estabelecimento da questão norteadora da pesquisa, busca na literatura, análise crítica dos estudos e síntese dos resultados obtidos em torno da Fitoterapia: Fitoterapia, Plantas medicinais, Fitoterápicos.

Inicialmente foram encontrados 32 artigos, que após triagem, foi aplicado o filtro referente a data de publicação que versaram entre 2011 e 2021, restaram 28 artigos. Os critérios de exclusão foram: artigos com conteúdo divergentes, repetidos, obsoletos e que não estivessem associadas as palavras-chaves, dessa forma, foram excluídos 2 artigos, restando 26 artigos. Após a leitura na integra, foram excluídos mais 6 artigos, restando 20 artigos, que foram usados nesta revisão integrativa, conforme dispostos na Figura 1.

Figura 1: Fluxograma da metodologia emprega na elaboração revisão integrativa.

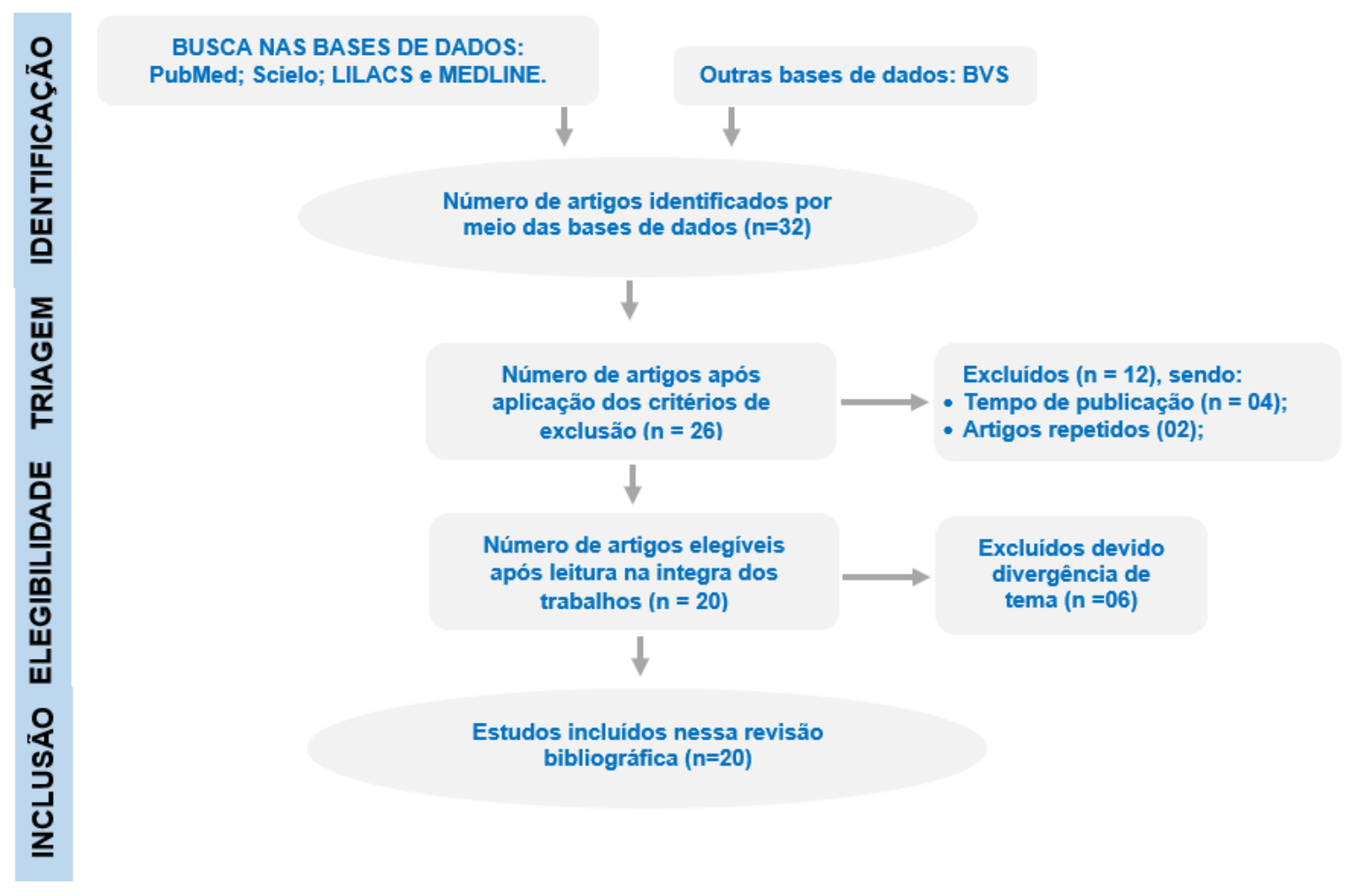

Fonte: Autores.

\section{Resultados e Discussão}

Todos os estudos estiveram relacionados com as palavras-chaves utilizadas como descritores. Na seleção da revisão integrativa foram identificados vários artigos relacionados à temática do presente estudo, sendo incluídos para revisão 20 (vinte) artigos, tendo em vista que atenderam aos critérios de inclusão previamente estabelecidos. Sendo importante ressaltar 
que os artigos contêm as variáveis estudadas no período de 2011 a 2021, conforme podem ser observados no Quadro 1, temos a análise dos principais resultados de cada artigo.

Quadro 1: Quadro sinóptico abordando os autores com o os principais resultados de seus artigos no âmbito da fitoterapia.

\begin{tabular}{|c|c|c|c|c|}
\hline Autor & Título & Local & Ano & Principais Resultados \\
\hline Abílio, G. M. F & Plantas Medicinais & Paraíba & 2011 & $\begin{array}{c}\text { Os resultados demonstram que apesar da importância } \\
\text { do uso das plantas medicinais, poucos são os estudos } \\
\text { que enfatizam a utilização dessas plantas. }\end{array}$ \\
\hline Badke M. R et al. & $\begin{array}{l}\text { Saberes e práticas populares de } \\
\text { cuidado em saúde com o uso de } \\
\text { plantas medicinais. }\end{array}$ & $\begin{array}{l}\text { Rio Grande do } \\
\text { Sul }\end{array}$ & 2012 & $\begin{array}{c}\text { Os resultados deste estudo demonstraram os } \\
\text { benéficos das plantas medicinais nativas e exóticas. }\end{array}$ \\
\hline Barquete, C. C. et al. & $\begin{array}{l}\text { Fitoterápicos amazônicos: copaíba } \\
\text { no tratamento de feridas cutâneas. }\end{array}$ & Acre & 2017 & $\begin{array}{l}\text { A importância do cultivo e estudos das plantas } \\
\text { medicinais usadas como ação cicatrizantes em feridas }\end{array}$ \\
\hline Bochner, R. & $\begin{array}{l}\text { Problemas associados ao uso de } \\
\text { plantas medicinais comercializadas } \\
\text { no Mercadão de Madureira, } \\
\text { município do Rio de Janeiro. }\end{array}$ & Rio de Janeiro & 2012 & $\begin{array}{l}\text { Os resultados mostram as plantas medicinais são } \\
\text { muito utilizadas, porém deve-se ter cuidados no } \\
\text { armazenamento e secagem para evitar contaminação. }\end{array}$ \\
\hline Carvalho, D. S. & $\begin{array}{l}\text { Preservação dos saberes tradicionais } \\
\text { assentamento São Francisco, de } \\
\text { plantas medicinais no Canutama, } \\
\text { Amazonas. } \\
\end{array}$ & Humaitá & 2019 & $\begin{array}{l}\text { Os resultados demonstram que a utilização de plantas } \\
\text { para o tratamento de doenças e benefícios à saúde } \\
\text { constitui-se como uma alternativa socialmente viável } \\
\text { e economicamente acessível. }\end{array}$ \\
\hline Costa, J. B. S. & $\begin{array}{l}\text { Um estudo etnobotanico sobre } \\
\text { plantas medicinais com base nos } \\
\text { saberes de um grupo de alunos da } \\
\text { educação de jovens e adultos. }\end{array}$ & Campina Grande & 2017 & $\begin{array}{l}\text { Os resultados demonstram que através das narrativas, } \\
\text { foi possível identificar de onde foram adquiridos os } \\
\text { conhecimentos etnobotanicos, como são utilizados } \\
\text { em suas práticas e para que fins as plantas medicinais } \\
\text { são usadas por estes alunos. }\end{array}$ \\
\hline $\begin{array}{l}\text { Dantas, J.I.M \& Torres, } \\
\text { A.M. }\end{array}$ & $\begin{array}{l}\text { A abordagem etnobotanica na } \\
\text { pesquisa de plantas medicinais em } \\
\text { uma comunidade rural do sertão } \\
\text { alagoano. }\end{array}$ & Alagoas & 2019 & $\begin{array}{l}\text { Os resultados apresentam que há uma grande } \\
\text { variedade de doenças tratadas por determinadas } \\
\text { plantas, desde doenças respiratórias, digestórias, } \\
\text { cardíacas e entre outras. Em relação ao uso de } \\
\text { determinadas plantas no tratamento de doenças, as } \\
\text { folhas e as cascas das plantas, foram o principal meio } \\
\text { indicado. }\end{array}$ \\
\hline Evangelista, S. S. et al. & $\begin{array}{l}\text { Fitoterápicos na odontologia: estudo } \\
\text { etnobotanico na cidade de Manaus. }\end{array}$ & Manaus & 2013 & $\begin{array}{c}\text { Os resultados mostram que existem pelo ao menos } 3 \\
\text { plantas medicinais sendo comercializadas na cidade } \\
\text { de Manaus para fins odontológicos. Mas são } \\
\text { utilizadas de maneira empírica. }\end{array}$ \\
\hline Feitosa, M. H. A. et al. & $\begin{array}{l}\text { Inserção do Conteúdo Fitoterapia em } \\
\text { Cursos da Área de Saúde. }\end{array}$ & $\begin{array}{l}\text { Montes Claros } \\
\text { MG }\end{array}$ & 2016 & $\begin{array}{l}\text { Os resultados são favoráveis à inserção do conteúdo } \\
\text { fitoterápico na grade curricular, embora ainda não } \\
\text { tendo conhecimentos da Política Nacional de Práticas } \\
\text { integrativas e Complementares. }\end{array}$ \\
\hline Lima A. R. A et al. & $\begin{array}{l}\text { Ações de mulheres agricultoras no } \\
\text { cuidado familiar: uso de plantas } \\
\text { medicinais no sul do brasil }\end{array}$ & Pelotas & 2014 & $\begin{array}{l}\text { O estudo demonstra a importância do uso de plantas } \\
\text { medicinais para curar dores e enfermidades das } \\
\text { mulheres residentes em Silves. }\end{array}$ \\
\hline $\begin{array}{l}\text { Linhares J.F.P } \\
\text { et. al. }\end{array}$ & $\begin{array}{l}\text { Etnobotanica das principais plantas } \\
\text { medicinais comercializadas em } \\
\text { feiras e mercados de São Luís, } \\
\text { Estado do Maranhão. } \\
\end{array}$ & Maranhão & 2014 & $\begin{array}{c}\text { Os resultados deste trabalho demonstraram que } \\
\text { Amazônia tem mais de } 10 \text { mil plantas com potencial } \\
\text { medicinal. }\end{array}$ \\
\hline Neto, F. R. G. et al. & $\begin{array}{l}\text { Estudo Etnobotanico de plantas } \\
\text { medicinais utilizadas pela } \\
\text { Comunidade do Sisal no } \\
\text { município de Catu, Bahia, Brasil. }\end{array}$ & Bahia & 2014 & $\begin{array}{c}\text { Os resultados despertam a atenção sobre a } \\
\text { necessidade da conservação da nossa flora e da } \\
\text { imediata aprovação de uma legislação específica que } \\
\text { assegure a proteção ao conhecimento tradicional do } \\
\text { povo indígenal }\end{array}$ \\
\hline Peckolt, T. & $\begin{array}{l}\text { História das plantas medicinais e } \\
\text { uteis do Brasil. }\end{array}$ & Belo Horizonte & 2016 & $\begin{array}{l}\text { Os resultados deste estudo demonstraram vários } \\
\text { estudos sobre a história das plantas medicinais do } \\
\text { Brasil. }\end{array}$ \\
\hline $\begin{array}{l}\text { Pedroso, R. S.; Andrade, } \\
\text { G.; Pires, R. H }\end{array}$ & $\begin{array}{l}\text { Mitos e verdades no uso de plantas } \\
\text { medicinais em uma comunidade } \\
\text { escolar. }\end{array}$ & Rio de Janeiro & 2021 & $\begin{array}{l}\text { Os resultados demonstram o uso racional das plantas } \\
\text { medicinais, e sua utilidade científica das práticas } \\
\text { populares e tradicionais de saúde, propondo a sua } \\
\text { associação à medicina moderna. }\end{array}$ \\
\hline Saltos, R.V.A et al. & $\begin{array}{l}\text { Uso de plantas medicinais por } \\
\text { populações rurais da província de } \\
\text { Pastaza, na Amazônia equatoriana. }\end{array}$ & Pastaza & 2016 & $\begin{array}{c}\text { Os resultados despertam a atenção sobre a } \\
\text { importância do uso medicinal das plantas da região } \\
\text { amazônica. }\end{array}$ \\
\hline $\begin{array}{l}\text { Santos, M. G.; Carvalho, } \\
\text { A. C. B }\end{array}$ & $\begin{array}{l}\text { Plantas medicinais: saberes } \\
\text { tradicionais e o sistema de saúde }\end{array}$ & Rio de janeiro & 2018 & $\begin{array}{c}\text { Os resultados mostram que existe um rico conjunto } \\
\text { de informações sobre plantas medicinais e que são } \\
\text { um excelente recurso terapêutico. }\end{array}$ \\
\hline $\begin{array}{l}\text { Silva, A. C; Lobato, F. } \\
\text { H. S e Ravena-Canete, } \\
\text { V. }\end{array}$ & $\begin{array}{c}\text { Plantas medicinais e seus usos em um } \\
\text { quilombo amazônico: o caso da } \\
\text { comunidade quilombola do Abacatal, } \\
\text { Ananindeua (PA) }\end{array}$ & Pará & 2019 & $\begin{array}{l}\text { Os resultados demonstram que apesar da importância } \\
\text { do uso das plantas medicinais, poucos são os estudos } \\
\text { que enfatizam a utilização dessas plantas, porém sua } \\
\text { utilização é de grande importância, principalmente }\end{array}$ \\
\hline
\end{tabular}




\begin{tabular}{|c|c|c|c|c|}
\hline & & & & pelas comunidades carentes. \\
\hline $\begin{array}{l}\text { Vásquez, S.P.L; } \\
\text { Mendonça, M.S; Noda. } \\
\text { S.N. }\end{array}$ & $\begin{array}{l}\text { Etnobotanica de plantas medicinais } \\
\text { em comunidades ribeirinhas do } \\
\text { município de Manacapuru, } \\
\text { Amazonas, Brasil. }\end{array}$ & Manacapuru & 2014 & $\begin{array}{c}\text { Os resultados demonstram que as Plantas medicinais } \\
\text { da Amazônia são de grande importância em } \\
\text { tratamentos de variadas doenças e os moradores } \\
\text { fazem uso como forma de tratar as doenças mais } \\
\text { frequentes. }\end{array}$ \\
\hline Vieira, S. C. H. V et al. & $\begin{array}{l}\text { Levantamento de fitoterápicos } \\
\text { manipulados em farmácias } \\
\text { magistrais de Dourados-MS } \\
\end{array}$ & Dourados & 2010 & $\begin{array}{l}\text { Os resultados deste trabalho demonstram } \\
\text { estritamente a visão científica e multidisciplinar de } \\
\text { especialistas acerca dos benefícios da fitoterapia. }\end{array}$ \\
\hline Zeni a..l...b et al. & $\begin{array}{l}\text { Utilização de plantas medicinais } \\
\text { como remédio caseiro na atenção } \\
\text { primária em Blumenau, Santa } \\
\text { Catarina, Brasil. }\end{array}$ & Santa Catarina & 2017 & $\begin{array}{c}\text { Os resultados mostram que esse potencial uso das } \\
\text { plantas medicinais como uso terapêutico e os } \\
\text { benefícios, como também a garantia da utilização de } \\
\text { forma adequada. }\end{array}$ \\
\hline
\end{tabular}

Fonte: Autores.

As categorias identificadas nos artigos após a análise dos principais resultados foram a análise crítico-reflexiva a respeito da fitoterapia com plantas nativas da região amazônica.

$\mathrm{Na}$ análise observou-se que os estudos apresentaram resultados semelhantes, no que se refere ao uso de plantas medicinais como sendo uma fonte muito importante e bastante utilizado para a manutenção da saúde de muitas comunidades carentes, pois a utilização de plantas para o tratamento de doenças e benefícios à saúde constitui-se como uma alternativa socialmente viável e economicamente acessível (Vásquez et al., 2014).

Dessa forma, conforme os descritos na literatura no que tange os principais benefícios do uso de medicamentos fitoterápicos, foi possível montar um quadro informativo (Quadro 2), contendo algumas plantas nativas da região amazônica com ação farmacológica, que com a devida utilização correta podem trazer cura e bem estar, consideradas culturalmente importantes nas comunidades, e merecem atenção especial para futuros estudos farmacológicos para que o conhecimento popular respaldado pelo conhecimento científico contribuam para o uso racional das plantas medicinais e conscientização da importância de conservá-las (Bochner, 2012; Dantas \& Torres, 2019).

Quadro 2: Demonstração das principais indicações do uso de plantas medicinais nativas da Amazônia.

\begin{tabular}{|c|c|c|c|}
\hline Nome popular & Nome científico & Modo de preparo & Indicações de uso \\
\hline Andiroba & Carapa guianensis & Decocção & $\begin{array}{l}\text { Possui propriedades anti-inflamatórias, antissépticas e cicatrizantes, } \\
\text { podendo ser utilizada no tratamento contra vermes, doenças de } \\
\text { pele, febre e inflamações. }\end{array}$ \\
\hline Camapu & Physalis angulata & Infusão & $\begin{array}{l}\text { Serve para tratar doenças neurodegenerativas, diminuir o colesterol } \\
\text { e fortalecer a imunidade. }\end{array}$ \\
\hline Carqueja & Baccharis trimera & Infusão & $\begin{array}{l}\text { Serve para regular a pressão arterial e os níveis de açúcar no } \\
\text { sangue, além de ajudar a fortalecer o sistema imunológico. }\end{array}$ \\
\hline Copaíba & $\begin{array}{l}\text { Copaifera } \\
\text { langsdorffii }\end{array}$ & Infusão & $\begin{array}{l}\text { Serve para tratamento de feridas cutâneas, possui propriedades anti- } \\
\text { inflamatórias, cicatrizantes e antisséptica. }\end{array}$ \\
\hline Elixir Parigórico & Ocimum seloi Benth & Infusão & Serve como antidiarreico, antiespasmódico e anti-inflamatório. \\
\hline Guaraná & Paullinia cupana & Medicinal & $\begin{array}{l}\text { Pode ser utilizado para aliviar os sintomas de hemorroidas, } \\
\text { enxaqueca, cólicas e ajuda na redução de peso, pois aumenta o } \\
\text { metabolismo da gordura. Esta planta tem algumas propriedades } \\
\text { semelhantes ao chá verde, principalmente por ser rico em } \\
\text { catequinas, que são substâncias antioxidantes. }\end{array}$ \\
\hline Jambu ou Agrião da Amazônia & Acmella oleracea & Decocção & $\begin{array}{l}\text { Possui propriedades anestésica local, laxante, diurético, antifúngica, } \\
\text { antiviral, afrodisíaca, antioxidante, rica em fibras e cálcio. }\end{array}$ \\
\hline Mutuquinha & Justicia pectoralis & Infusão & $\begin{array}{l}\text { Possui propriedades analgésica, antiespasmódica, anti-inflamatória, } \\
\text { antipirética, expectorante e broncodilatadora. }\end{array}$ \\
\hline Marupazinho & Eleutherine bulbosa & Decocção & Serve para combater problemas gastrointestinais. \\
\hline Terramicina & $\begin{array}{l}\text { Anthernanthera } \\
\text { brasiliana }\end{array}$ & Decocção & Possui propriedades para combater dores, trato respiratório e tosse. \\
\hline
\end{tabular}

Fonte: Autores. 
Desse modo, de acordo com as observações dos estudos a fitoterapia das plantas regionais deve-se ao fato dessas inúmeras espécies cultivadas apresentarem uma produção constante, proporcionando remédios variados que complementam a saúde, a preferência por ser de fácil acesso, baixo custo e de efeitos colaterais reduzidos. (Peckolt, 2016; Lorenzi, 2018).

É importante salientar que praticamente todas as plantas e elementos da natureza possuem princípios ativos que podem ser utilizados como medicamentos, sendo a extração dos princípios ativos dessas plantas, fundamental para sua utilização na saúde. Pesquisas feitas em geral, nascem a partir do interesse despertado pelo conhecimento e utilização dos povos tradicionais, assim o uso das plantas medicinais deve ser feito com muito cuidado e atenção em relação às doses e efeitos individuais, pois cada organismo reage de uma maneira aos princípios ativos das plantas, portanto é importante observar eventuais reações adversas, e devido à ausência de maiores estudos é sempre recomendado evitar o uso de ervas durante a gravidez, amamentação e em crianças menores de 5 anos (Neto et al; Lima et al., 2014).

Com isso, a utilização e consumo destas plantas medicinais é reconhecida atualmente pela Organização Mundial da Saúde - OMS, devido ao conhecimento empírico secular sobre a ação desses vegetais reconhecido por diversos grupos étnicos, resultando na descoberta da medicina tradicional. No Brasil, além dos conhecimentos tradicionais indígenas, as contribuições trazidas de outros povos tiveram uma importância significativa no surgimento da medicina popular rica baseada na utilização da biodiversidade vegetal nacionais, promovendo uma infinidade de diferentes espécies de plantas medicinais em prol à saúde humana (Vásquez et al., 2014).

A exemplo das plantas medicinais, o crescimento do mercado de fitoterápicos cresceu devido ao aumento da busca por ativos naturais pela indústria de medicamentos e a expansão da indústria de cosméticos e perfumes. A implementação da fitoterapia no âmbito dos serviços de Atenção Básica à Saúde (ABS) no Brasil teve início em 1988, principalmente após a descentralização, incentivo da participação popular e crescimento da autonomia municipal na área da saúde (Neto et al; Lima et al., 2014). Por esse motivo, o Ministério da Saúde, propôs o objetivo de ampliar o acesso da população aos serviços relacionados a fitoterapia, aprovando a Política Nacional de Práticas Integrativas e Complementares em Saúde por meio da Portaria $n^{\circ} .971$, de 03 de maio de 2006 e a Política Nacional de Plantas Medicinais e Fitoterápico (PNPMF) pelo Decreto 5813, 26 de junho de 2006.

Dessa forma, o governo buscou por meio de políticas publicar fornecer bases legais para a promoção da fitoterapia racional, valorizando o conhecimento tradicional e o uso de plantas medicinais como forma de prevenir, atenuar e curar patologias (Silva et al., 2019).

\section{Considerações Finais}

Diante dessa revisão integrativa da literatura é possível despertar o leitor a buscar a cura por meio dos segredos milenares da Amazônia, relacionados ao uso tradicional das plantas medicinais. Mostrando que o uso de plantas medicinais ainda é um importante recurso utilizado para a manutenção da saúde de muitas comunidades carentes, onde são utilizadas para tratamentos comuns de saúde, tendo em vista que o custo é mais acessível à população, em comparação aos medicamentos obtidos por síntese química, que são em geral mais caros, devido às patentes tecnológicas envolvidas.

Além disso, o uso de plantas medicinais, pode ser um importante aliado na promoção da saúde na atenção primária. É importante ressaltar que diversos estudos têm sido desenvolvidos para modificar o conhecimento empírico com o intuito de comprovar a ação de fitoterápicos, lançando assim bases para a fitoterapia racional e uma promoção mais seguro no uso de fitoterápicos e plantas medicinais.

Sendo assim, é de grande importância para a comunidade científica que se continue a investigar e estudar as propriedades terapêuticas das plantas aromáticas e dessa forma que possamos oferecer mais opções de tratamento acessíveis 
para a população, contribuindo forma direta ou indireta para o fortalecimento e manutenção da fitoterapia e o conhecimento tradicional acerca do uso de plantas medicinais.

\section{Referências}

Abílio, G. M. F. (2011). Plantas Medicinais. Bananeiras, Editora Universitária/UFPB. 6(7), 4. 7766-8477.

Badke M. R et al. (2012). Saberes e práticas populares de cuidado em saúde com o uso de plantas medicinais, 21(2), 363-70. https://doi.org/10.1590/S010407072012000200014 .

Barquete, C.C. (2017). Fitoterápicos amazônicos: copaíba no tratamento de feridas cutâneas. Rev. Conhecer Cientifico, 14(26), 361.

Bochner, R. (2012). Problemas associados ao uso de plantas medicinais comercializadas no Mercadão de Madureira, município do Rio de Janeiro,14(3), 537547. https://www1.ibb.unesp.br/Home/Departamentos/Botanica/RBPM.

Carvalho, D. S. (2019). Preservação dos saberes tradicionais assentamento São Francisco, de plantas medicinais no Canutama, Amazonas. Humaitá. Dissertação (Mestrado em Ciências Ambientais) - Universidade Federal do Amazonas. https://tede.ufam.edu.br/handle/tede/7350.

Costa, J. B. S. (2017). Um estudo etnobotanico sobre plantas medicinais com base nos saberes de um grupo de alunos da educação de jovens e adultos. Dissertação (Programa de Pós-Graduação em Ensino de Ciências e Educação Matemática - PPGECEM) - Universidade Estadual da Paraíba, Campina Grande. http://tede.bc.uepb.edu.br/jspui/handle/tede/2858.

Dantas, J. I. M., \& Torres, A. M. (2019). A abordagem etnobotanica na pesquisa de plantas medicinais em uma comunidade rural do sertão alagoano, 4(1), 3948. https://doi.org/10.17648/diversitas-journal-v4i1.663.

Evangelista, S. S., Sampaio, F. C., Parente, R. C., \& Bandeira, M. F. C. L. (2013). Fitoterápicos na odontologia: estudo etnobotanico na cidade de Manaus, 15(4), 513-519. https://doi.org/10.1590/S1516-05722013000400007.

Feitosa, M. H. A. et al. (2016). Inserção do Conteúdo Fitoterapia em Cursos da Área de Saúde. Revista Brasileira de Educação Médica [online], 40(2), 197203. https://doi.org/10.1590/1981-52712015v40n2e03092014.

Gaio, R., Carvalho, R. B., \& Simões, R. (2008). Métodos e técnicas de pesquisa: a metodologia em questão. In: Gaio, R. (org.). Metodologia de pesquisa e produção de conhecimento. Petrópolis, Vozes, 147-171.

Lima A. R. A et al. (2014). Ações de mulheres agricultoras no cuidado familiar: uso de plantas medicinais no sul do Brasil, 3(2), 365-72. https://doi.org/10.1590/0104-07072014004080012.

Linhares, J. F. P et al. (2014). Etnobotanica das principais plantas medicinais comercializadas em feiras e mercados de São Luís, Estado do Maranhão, 5(3), 39-46. http://dx.doi.org/10.5123/S2176-62232014000300005.

Lorenzi, H., \& Matos, F. J. A. (2018). Plantas Medicinais no Brasil: nativas e exóticas. (2a ed.), 544.

Neto, F. R. G., Almeida, G. S. S. A., Jesus, N.G., \& Fonseca, M. R. (2014). Estudo Etnobotanico de plantas medicinais utilizadas pela Comunidade do Sisal no município de Catu, Bahia, Brasil, 16(4), 856-865. https://doi.org/10.1590/1983-084X/11_207.

Peckolt, T. (2016). História das plantas medicinais e uteis do Brasil. 904.

Pedroso, R. S., Andrade, G., \& Pires, R. H. (2021). Plantas medicinais: uma abordagem sobre o uso seguro e racional, 31(2), 310218. https://doi.org/10.15 90/S0103-73312021310218.

Saltos, R. V. A et al. (2016). Uso de plantas medicinais por populações rurais da província de Pastaza, na Amazônia equatoriana, 46(4), 355-366. https://doi.o $\mathrm{rg} / 10.1590 / 1809-4392201600305$.

Santos, M. G., \& Carvalho, A. C. B. (2018). Plantas medicinais: saberes tradicionais e o sistema de saúde, 4, 71-99. https://doi.org/10.7476/9788 5751148 58.0006 .

Silva, A. C, Lobato, F. H. S, \& Ravena-Canete, V. (2019). Plantas medicinais e seus usos em um quilombo amazônico: o caso da comunidade Quilombola do Abacatal, Ananindeua,11(3), 113-136. http://dx.doi.org/10.26823/RevistadoNUFEN.vol11.nº3artigo6.

Silva, D. F., Lima, K. T., Bastos, G. N. T., Oliveira, J. A. R., do Nascimento, L. A. S., Costa, C. E. F., Filho, G. N. R., Concha, V. O. C., \& Passos, M. F. (2021). PCL/Andiroba Oil (Carapa guianensis Aubl.) Hybrid Film for Wound Healing Applications. Polymers (Basel), 14,13(10), 1591. https://doi.org/10.3390/polym13101591

Vásquez, S. P. L, Mendonça, M. S, \& Noda. S. N. (2014). Etnobotanica de plantas medicinais em comunidades ribeirinhas do Município de Manacapuru, Amazonas, Brasil, 44(4), 457 - 472. https://doi.org/10.1590/1809-4392201400423.

Vieira, S. C. H. et al. (2010) Levantamento de fitoterápicos manipulados em farmácias magistrais de Dourados-MS. Revista Brasileira de Farmacognosia, 20(1), 28-34. https://doi.org/10.1590/S0102-695X2010000100007.

Zeni, A. L. B., et al. (2017). Utilização de plantas medicinais como remédio caseiro na Atenção Primária em Blumenau, Santa Catarina, Brasil, 22(8), 27032712. https://doi.org/10.1590/1413-81232017228.18892015. 